\title{
Clinical practice patterns in indirect peroral cholangiopancreatoscopy: outcome of a European survey
}

\section{(ㄷ)(1) $\odot$}

\section{Authors}

Pauline M.C. Stassen ${ }^{1}$, Pieter Jan F. de Jonge ${ }^{1}$, George J.M. Webster ${ }^{2}$, Mark Ellrichmann ${ }^{3}$, Arno J. Dormann ${ }^{4}$, Marianne Udd $^{5}$, Marco J. Bruno ${ }^{1}$, Vincenzo Cennamo ${ }^{6}$, on behalf of the European Cholangioscopy Group, and the German Spyglass User Group

\section{Institutions}

1 Department of Gastroenterology and Hepatology, Erasmus MC University Medical Center Rotterdam, Rotterdam, The Netherlands

2 Department of Gastroenterology, University College London Hospitals, London, United Kingdom

3 Department of Interdisciplinary Endoscopy, Medical Department 1, University Hospital Schleswig-Holstein, Campus Kiel, Kiel, Germany

4 Department of Gastroenterology, GI Oncology, GI-ID, Nutritional Medicine, Kliniken der Stadt Köln, Köln, Germany

5 Department of Gastroenterological Surgery, University of Helsinki and Helsinki University Hospital, Helsinki, Finland

6 Gastroenterology and Interventional Endoscopy Unit, Local Health Authority of Bologna, Bologna, Italy

submitted 23.3.2021

accepted after revision 7.6.2021

\section{Bibliography}

Endosc Int Open 2021; 09: E1704-E1711

DOI 10.1055/a-1535-1458

ISSN 2364-3722

(C) 2021. The Author(s).

This is an open access article published by Thieme under the terms of the Creative Commons Attribution-NonDerivative-NonCommercial License, permitting copying and reproduction so long as the original work is given appropriate credit. Contents may not be used for commercial purposes, or adapted, remixed, transformed or built upon. (https://creativecommons.org/licenses/by-nc-nd/4.0/)

Georg Thieme Verlag KG, Rüdigerstraße 14,

70469 Stuttgart, Germany

\section{Corresponding author}

Pauline M.C. Stassen, MD, Department of Gastroenterology and Hepatology, Erasmus MC University Medical Center Rotterdam, Dr. Molewaterplein 40, 3050 GD Rotterdam, The Netherlands

Fax: +31-10-7030331

p.stassen@erasmusmc.nl $\circledast$ Supplementary material is available under https://doi.org/10.1055/a-1535-1458

\section{ABSTRACT}

Background and aims Indirect peroral cholangiopancreatoscopy (IPOC) is a relatively new diagnostic and therapeutic tool for biliopancreatic diseases. This international survey aimed to evaluate clinical practice patterns in IPOC among endoscopists in Europe.

Methods An online survey was developed comprising 66 questions on the use of IPOC. Questions were grouped into four domains. The survey was sent to 369 endoscopists who perform IPOC.

Results 86 respondents (23.3\%) from 21 different countries across Europe completed the survey. The main indications for cholangioscopy were determination of biliary strictures (85 [98.8\%]) and removal of common bile duct or intrahepatic duct stones (79 [91.9\%]), accounting for an estimated use of $40 \%$ (interquartile range [IQR] 25-50) and $40 \%$ (IQR 30-60), respectively, of all cases undergoing cholangioscopy. Pancreatoscopy was mainly used for removal of pancreatic duct stones (68/76 [89.5\%]), accounting for an estimated use of $76.5 \%$ (IQR 50-95) of all cases undergoing pancreatoscopy. Only $13 / 85$ respondents (15.3\%) had an institutional standardized protocol for targeted cholangioscopy-guided biopsy sampling. IPOC with lithotripsy was used as first-line treatment in selected patients with bile duct stones or pancreatic stones by $24 / 79$ (30.4\%) and 53/68 (77.9\%) respondents, respectively.

Conclusions This first European survey on the clinical practice of IPOC demonstrated wide variation in experience, indications, and techniques. These results emphasize the need for prospective studies and development of an international consensus guideline to standardize the practice and quality of IPOC. 


\section{Introduction}

Indirect peroral cholangiopancreatoscopy (IPOC) is a relatively new technique that was first described in the 1970 s, but with an increasing role in the management of biliopancreatic diseases over the past 15 years [1]. IPOC has enabled endoscopists to directly visualize the intraductal mucosa. Together with the development of dedicated accessories, this technology has led to an increased diagnostic and therapeutic capability, by assisting in the differentiation of malignant from benign lesions, taking targeted intraductal biopsies, and delineating intraductal tumors. Moreover, fragmentation of (difficult) bile duct or pancreatic duct stones can now be performed under direct visualization. The European Society of Gastrointestinal Endoscopy (ESGE) and the American Society for Gastrointestinal Endoscopy (ASGE) have provided technology reviews on intraductal biliopancreatic imaging [2,3], presenting an overview of the currently available techniques and instruments for intraductal imaging. Furthermore, diagnostic and therapeutic indications have been described based on the currently available literature. However, despite the technology reviews of the ESGE and ASGE, a clinical practice guideline or standardized protocol for IPOC is lacking. As IPOC is increasingly used in daily practice and technical improvements continue to be made, development of an international clinical guideline is crucial. Unfortunately, only a limited number of high quality studies are available in this field. As a result, current practice mainly relies on expert opinion and personal preference. In the process of developing a guideline, knowledge of current clinical practice in the performance of IPOC is necessary. Therefore, the aim of this survey was to evaluate the current clinical practice of IPOC in Europe, with regard to applied techniques and main indications.

\section{Methods}

\section{Study design}

A European online survey was conducted among endoscopists who perform cholangioscopy and/or pancreatoscopy. The survey was sent to members of the European Cholangioscopy Group (see Supplementary material), which is an international research group established in 2018, with 37 current members who are expert pancreaticobiliary endoscopists, from 10 different countries. In addition, the survey was sent to members of the German SpyGlass User Group (see Supplementary material), authors working in European hospitals who have published on IPOC in the past 5 years and for whom contact details were available online, and to SpyGlass users who had given consent to Boston Scientific Inc. (Marlborough, Massachusetts, USA) for commercial use of their contact details.

The survey questions were developed by the core study team (P.M.C.S., P.J.F.dJ., M.J.B., and V.C.), and were tested for content and clarity by a native English speaker (G.J.M.W.) and three other authors (M.E., A.J.D., and M.U). The survey consisted of 66 questions, divided into four domains:
1. experience in endoscopic retrograde cholangiopancreatography (ERCP), cholangioscopy, and pancreatoscopy;

2. equipment and technique;

3. indications for cholangioscopy; and

4. indications for pancreatoscopy.

An online survey was built using LimeSurvey, Version 2.06 LTS (LimeSurvey Project, Hamburg, Germany). The contents of this survey are provided in the supplementary Appendix. Invitations were sent via email in September 2019, with reminders sent in October and November 2019.

\section{Statistical analysis}

Only completed surveys were used for statistical analysis. Descriptive statistics were used to analyze the data, using median and interquartile range (IQR) for non-normally distributed continuous variables, and frequencies and percentages for categorical variables. The statistical analyses were performed using the statistical software package SPSS version 25 (IBM Corp, Armonk, New York, USA).

\section{Results}

The survey was sent to 369 endoscopists who perform cholangioscopy and/or pancreatoscopy. In total, 86 endoscopists (23.3\%) completed the survey, $25 \%$ of whom were affiliated to the European Cholangioscopy Group or German SpyGlass User Group. Responses were received from 21 countries and 71 different hospitals. Demographic details are shown in Table 1s.

\section{Endoscopic experience}

The majority of the respondents were very experienced in ERCP, with a lifetime experience of over 1000 procedures (68 [79.1\%]). Experience in IPOC was much more variable ( $\mathbf{F i g . 1}$ ), with a greater lifetime experience in cholangioscopy than in pancreatoscopy, and also a higher number of cholangioscopy procedures performed on average per year over the past 5 years compared with pancreatoscopy procedures. Experience in pancreatoscopy showed that $6 / 86(7.0 \%)$ had performed $>100$ procedures, but the majority of respondents (58/86 [67.4\%]) had performed no more than 25 pancreatoscopies.

\section{Equipment and technique}

The vast majority of respondents used the second-generation SpyGlass DS Visualization System (82 [95.3\%]). Compared with ERCP without IPOC, general anesthesia with endotracheal intubation was used more frequently than other sedation techniques for ERCP with IPOC. Antibiotic prophylaxis prior to or during cholangioscopy was routinely administered by most respondents (76.7\%). Intraprocedurally, access to the biliary tree and to the pancreatic duct (PD) was achieved via over-the-wire insertion by the majority of the respondents (60 [69.8\%] and $75[87.2 \%]$, respectively). The remaining respondents performed direct access by free-hand cannulation. These results are presented in $>$ Table $\mathbf{1}$. 


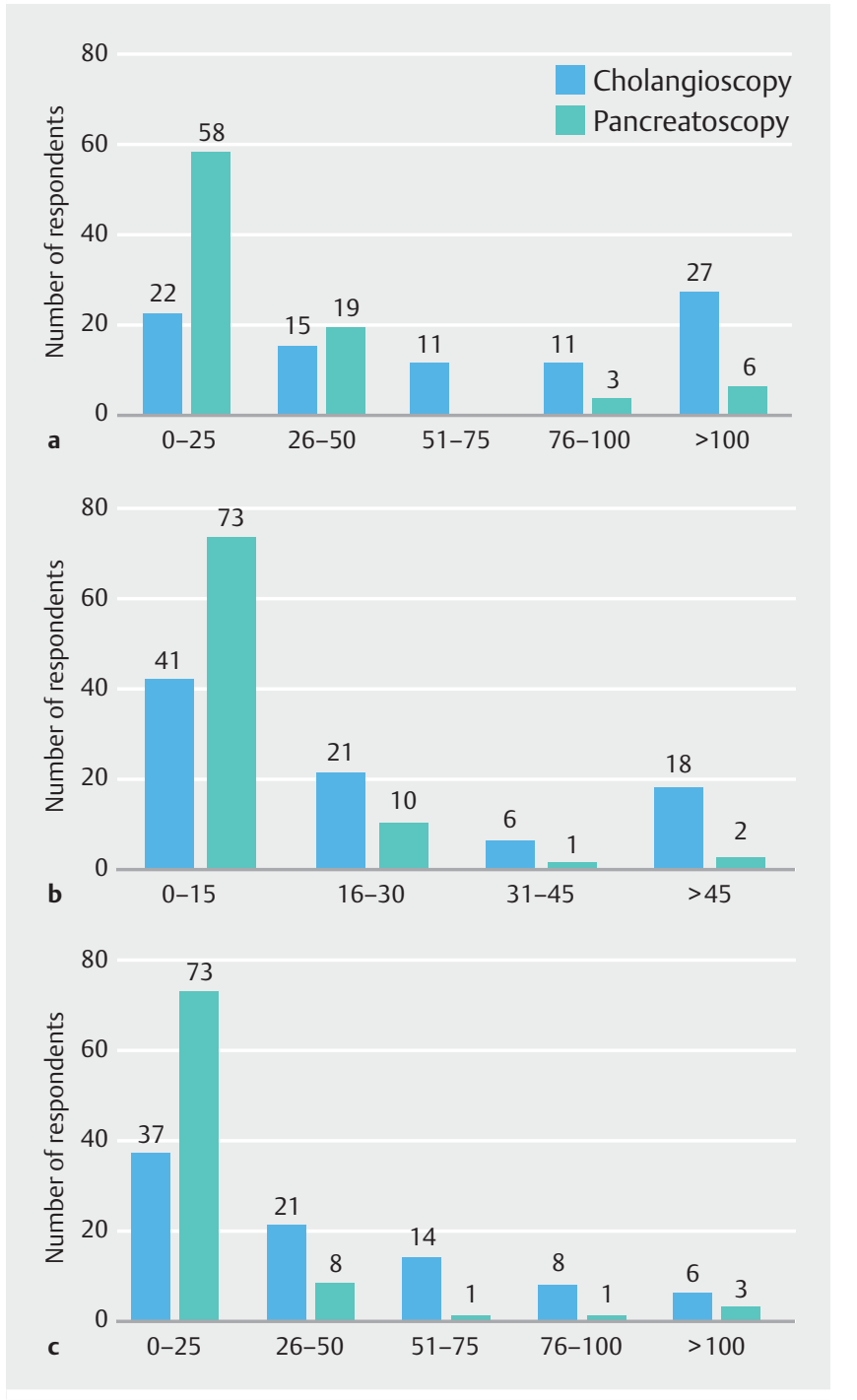

- Fig. 1 Experience in indirect peroral cholangiopancreatoscopy. a Total lifetime number of cholangioscopies and pancreatoscopies performed by the respondents. $\mathbf{b}$ Average number of cholangioscopies and pancreatoscopies performed by the respondents per year over the past 5 years. c Total number of cholangioscopies and pancreatoscopies performed per year in the hospitals of the respondents.

\section{Indications for cholangioscopy}

The three indications accounting for the most frequently performed procedures in daily clinical practice as reported by the respondents were:

1. removal of common bile duct $(\mathrm{CBD})$ or intrahepatic stones (40\% of procedures);

2. determination of biliary strictures ( $40 \%$ of procedures); and

3. delineation of known cholangiocarcinoma (CCA; $10 \%$ of procedures).

The results are shown in $>$ Table 2 .

\section{Bile duct stones}

In total, 79/86 respondents (91.9\%) used IPOC for removal of CBD and intrahepatic duct stones. The results are summarized in Table 2s. In the treatment strategy, only one-third of respondents (30.4\%) used IPOC with lithotripsy as first-line treatment without prior ERCP with conventional stone extraction techniques. In general, the mean number of IPOC procedures reported to be required to achieve stone clearance was 1 or 2 ( $46.8 \%$ and $48.1 \%$, respectively). Two types of lithotripsy tools were available to respondents: electrohydraulic lithotripsy (EHL) by $64.6 \%$ and laser lithotripsy by $20.3 \%$ of users. Both tools were used by $15.2 \%$ of users.

IPOC for bile duct stone removal was most frequently used for stones located in the proximal CBD (93.7\%) and in the hilum $(92.4 \%)$, but was also used for stones located in the distal CBD (73.4\%), left and right intrahepatic ducts $(86.1 \%$ and $88.6 \%$, respectively), and the cystic duct (64.6\%). In cases with failed bile duct stone removal using IPOC with lithotripsy, this was mostly reported to be due to difficult fragmentation using $\mathrm{EHL}$ or laser lithotripsy (57.0\%). Other frequently reported reasons included a stricture distal to the stone $(48.1 \%)$, difficult stone location $(46.8 \%)$, or the size of the stone $(50.6 \%)$. Difficult stone locations were mostly the intrahepatic left and right ducts $(75.7 \%$ and $64.9 \%$, respectively).

\section{Determination of biliary strictures}

In total, 85/86 respondents (98.8\%) used IPOC for determination of biliary strictures. For optimal intraductal visualization, the vast majority $(92.9 \%)$ used white light and only a minority (7.1\%) used white light in combination with chromoendoscopy (with vital staining). All respondents reported taking targeted biopsies under cholangioscopic visualization; however, only $63.5 \%$ of respondents reported taking cholangioscopic targeted biopsies routinely in all patients. The remaining respondents did this in at least $50 \%$ of patients $(29.4 \%)$ or in less than $50 \%$ of patients (7.1\%). Furthermore, even though all respondents reported taking cholangioscopic targeted biopsies, only $15.3 \%$ reported having an institutional protocol available for targeted cholangioscopy-guided tissue sampling. Nevertheless, the vast majority of respondents took at least three biopsies (96.5\%) and the majority (82.4\%) took bite-on-bite biopsies from the identified lesions. After cholangioscopy-guided biopsies had been taken, about half of the respondents (48.2 \%) performed additional fluoroscopy-guided brush cytology. Finally, rapid onsite evaluation of touch imprint cytology (ROSETIC) or fluorescence in situ hybridization analysis was available to only a minority of respondents $(2.4 \%$ and $9.4 \%$, respectively). The results are shown in Table $3 \mathrm{~s}$.

\section{Delineation of CCA}

IPOC for the preoperative delineation of CCA was used by $45 / 86$ respondents (52.3\%). A total of 42 respondents (93.3\%) used IPOC with delineation as part of the preoperative diagnostic work-up protocol, all prior to surgery and not during surgery, with $69.0 \%$ relying on both visual characteristics and mapping biopsies. 
- Table 1 Equipment and techniques used by respondents $(n=86)$.

\section{n (\%)}

The main type of cholangiopancreatoscope used (multiple options possible)

- SpyGlass Direct Visualization System (first-genera-
tion) ${ }^{1}$

$11(12.8)$

The SpyGlass DS Direct Visualization System ${ }^{1}$

82 (95.3)

- $\mathrm{CHF}-\mathrm{BP} 30^{2}$

- Polyscope ${ }^{3}$

Number of operators

\begin{tabular}{l|l|}
\hline - Single & $76(88.4)$ \\
\hline - Double & $10(11.6)$ \\
\hline
\end{tabular}

Preferred patient position during IPOC

- Supine $32(37.2)$

- Prone $40(46.5)$

- Left lateral 14 (16.3)

The most common type of sedation used for ERCP without IPOC

\begin{tabular}{|l|l|}
\hline - Conscious sedation & $24(27.9)$ \\
\hline - Nurse-administered propofol sedation & $23(26.7)$ \\
\hline - Propofol administered by anesthesiologist & $18(20.9)$ \\
\hline - General anesthesia with endotracheal intubation & $21(24.4)$ \\
\hline
\end{tabular}

The most common type of sedation used for ERCP with IPOC

\begin{tabular}{|c|c|}
\hline - Conscious sedation & $6(7.0)$ \\
\hline - Nurse-administered propofol sedation & $21(24.4)$ \\
\hline - Propofol administered by anesthesiologist & $24(27.9)$ \\
\hline - General anesthesia with endotracheal intubation & $35(40.7)$ \\
\hline \multicolumn{2}{|l|}{ IPOC schedule } \\
\hline - Outpatient procedure, discharge on the same day & $30(34.9)$ \\
\hline - Inpatient procedure, at least 1 night hospital stay & $56(65.1)$ \\
\hline $\begin{array}{l}\text { - Routine prophylactic antibiotics in patients under- } \\
\text { going IPOC }\end{array}$ & $66(76.7)$ \\
\hline
\end{tabular}

Access to the biliary tree

\begin{tabular}{|l|l|}
\hline . Over-the-wire insertion & $60(69.8)$ \\
\hline . Direct access & $26(30.2)$ \\
\hline
\end{tabular}

Access to the pancreatic duct

\begin{tabular}{|l|l|}
\hline - Over-the-wire insertion & $75(87.2)$ \\
\hline
\end{tabular}

- Direct access

$11(12.8)$

Type of medium used to improve visualization

- Sterile saline $76(88.4)$

- Water

$7(8.1)$

- Carbon dioxide

$3(3.5)$
- Table 1 (Continuation)

IPOC, indirect peroral cholangiopancreatoscopy; ERCP, endoscopic retrograde cholangiopancreatography.

${ }^{1}$ Boston Scientific, Marlborough, Massachusetts, USA.

2 Olympus, Tokyo, Japan.

${ }^{3}$ PolyDiagnost GmbH, Hallbergmoos, Germany.

With regard to clinical relevance, the majority of respondents reported that only in a small proportion of patients was the surgical approach altered by cholangioscopic findings preoperatively: in $0-20 \%$ of the patients according to $31.1 \%$ and in $21 \%-40 \%$ of the patients according to $42.2 \%$ of respondents. The results are presented in Table $4 s$.

\section{Indications for pancreatoscopy}

A total of 10 respondents recorded that they did not perform pancreatoscopy, resulting in $88.4 \%$ of respondents $(n=76)$ performing pancreatoscopy. The three indications accounting for the most frequently performed procedures in daily clinical practice were:

1. removal of PD stones (76.5\% of procedures);

2. determination of pancreatic strictures (30\% of procedures); and

3. delineation of intraductal papillary mucinous neoplasms (IPMN; $20 \%$ of procedures).

\section{These results are shown in $>$ Table 3 .}

\section{Pancreatic duct stones}

In total, 68/76 respondents (89.5\%) used IPOC for removal of PD stones. In the treatment algorithm of PD stones, $77.9 \%$ used IPOC with lithotripsy as first-line treatment, without prior extracorporeal shock wave lithotripsy (ESWL). However, 89.7\% of users first performed conventional ERCP with sphincterotomy before performing IPOC lithotripsy. For PD stone removal, the majority of respondents used EHL (70.6\%), 17.6\% used laser lithotripsy, and $11.8 \%$ used both tools for PD stone fragmentation. IPOC for PD stone removal was mostly used for stones located in the head or the neck of the pancreas $(97.1 \%$ and $75.0 \%$, respectively). Half of the respondents (50.0\%) reported successful PD stone removal in a single IPOC session. Where multiple sessions were required to achieve complete stone removal, this was due to a large stone size (67.6\%), difficult stone location (52.9\%), a stricture distal to the stone(s) (52.9\%), or difficult stone fragmentation with EHL or laser lithotripsy (67.6\%). Finally, after IPOC with lithotripsy, $89.7 \%$ reported placing a PD stent to provide drainage of pancreatic fluid. All responses are shown in Table 5s.

\section{Delineation of IPMN}

IPOC for the delineation of main duct IPMN was used by 35/76 respondents (46.1\%). Similarly to the use of IPOC in the preoperative work-up of CCA, IPOC was part of the preoperative diagnostic work-up of IPMN by $80.0 \%$ of respondents, with $71.4 \%$ relying on both visual characteristics and mapping biopsies for determining the extent of IPMN. The surgical approach was altered in less than half of the patients: in only $0-20 \%$ of the pa- 
- Table 2 Indications for cholangioscopy $(n=86)$.

\begin{tabular}{|l|l|l|}
\hline Biliary indication & Respondents, $\mathbf{n}(\%)$ & Use in daily clinical practice, median, (IQR), \% \\
\hline Determination of biliary strictures & $85(98.8)$ & $40(25-50)$ \\
\hline Removal of CBD or intrahepatic duct stones & $79(91.9)$ & $40(30-60)$ \\
\hline Selective segment cannulation & $57(66.3)$ & $5(3.5-10)$ \\
\hline Delineation of CCA & $45(52.3)$ & $10(5-17.5)$ \\
\hline Removal of cystic duct stones & $45(52.3)$ & $5(2-5)$ \\
\hline Removal of foreign bodies within the bile duct & $43(50.0)$ & $5(3-8)$ \\
\hline Stricture negotiation & $38(44.2)$ & $3(1-5)$ \\
\hline Recanalization of postoperative biliary disconnection & $23(26.7)$ & $1(1-3)$ \\
\hline Transpapillary gallbladder drainage for acute cholecystitis & $5(5.8)$ & 2 \\
\hline Access to biliodigestive anastomosis after Whipple's resection & $1(1.2)$ & 38 \\
\hline Transgastric access to the bile duct & $1(1.2)$ & \\
\hline IQR, interquartile range; CBD, common bile duct; CCA, cholangiocarcinoma. & & \\
\hline
\end{tabular}

- Table 3 Indications for pancreatoscopy $(n=76)$.

\begin{tabular}{|l|l|l|}
\hline Pancreatic indication & Respondents, $\mathbf{n}(\%)$ & Use in daily clinical practice, median, (IQR), \% \\
\hline Removal of pancreatic duct stones & $68(89.5)$ & $76.5(50-95)$ \\
\hline Determination of indeterminate pancreatic strictures & $41(53.9)$ & $30(15-40)$ \\
\hline Delineation of IPMN & $35(46.1)$ & $20(10-40)$ \\
\hline Removal of foreign bodies within the pancreatic duct & $27(35.5)$ & $5(5-20)$ \\
\hline Dilatation of the Wirsung duct & $1(1.3)$ & 6 \\
\hline IQR, interquartile range; IPMN, intraductal papillary mucinous neoplasm. & & \\
\hline
\end{tabular}

tients according to $42.9 \%$ and in $21 \%-40 \%$ of the patients according to $46.4 \%$ of respondents. These results are presented in Table 6s.

\section{Discussion}

This is the first European survey to investigate current clinical practice patterns of indirect IPOC among endoscopists experienced in ERCP. The results showed that there was wide variation in both experience and use of IPOC. IPOC was most frequently used for biliary indications, mainly evaluation of biliary strictures and stone removal, which together accounted for a median of $80.0 \%$ of the indications that IPOC was used for in daily clinical practice.

According to this survey, the evaluation of biliary strictures was one of the main indications for cholangioscopy. Previous studies have shown very good results regarding the diagnostic accuracy of visual characteristics for determining the etiology of strictures, but considerable interobserver variation exists among endoscopists [4,5]. Morphological features of malignant and inflammatory disease may overlap significantly and not be exclusive or clearly discriminative, even for experienced endoscopists. As such, the widely used phrase "tissue is the is- sue" remains central. Kalaitzakis et al. showed that obtaining more than four biopsies compared with fewer than four biopsies resulted in a higher adequacy rate of the tissue samples ( $90 \%$ vs. 64\%; $P=0.037$ ) [6]. In addition, Gerges et al. showed that an accuracy rate of $66 \%$ can be achieved by taking at least three biopsies [7]. In the current survey, we found that only $15.3 \%$ of respondents had an institutional protocol for taking cholangioscopy-guided biopsies. However, 96.5\% took at least three biopsies, which should increase accuracy rates of the specimen samples according to the literature. Furthermore, techniques for optimizing tissue yield vary widely, with some endoscopists performing bite-on-bite biopsies, and others obtaining an additional brushing after IPOC-directed biopsies. Very few respondents performed ROSE-TIC analysis. This technique has shown promising results in two studies, in which ROSE-TIC used for the diagnosis of indeterminate biliary strictures and pancreaticobiliary lesions showed sensitivity and specificity rates of $97 \%-100 \%$ and $88 \%-89 \%$, respectively $[8,9]$. Optimizing visually directed pathological sampling may be the most important current task for cholangioscopic assessment of biliary strictures. Prospective studies are needed in order to inform the development of an international cholangioscopy-guided biopsy protocol aimed at improving diagnostic accuracy rates. 
An interesting finding of this survey was the relatively common use of IPOC in clinical practice for preoperative determination of the extent ("mapping") of IPMN and/or CCA. The timing in clinical work-up for both indications is debatable. To the best of our knowledge, there is no guideline available recommending the timing and use of cholangioscopy in the preoperative work-up of CCA. For IPMN, the European Study Group of Cystic Tumours of the Pancreas has written an evidence-based guideline on pancreatic cystic neoplasms and recommended that pancreatoscopy may be used in selected cases to provide information on the location and extent of main duct IPMN, with higher accuracy rates reported for main duct IPMN compared with branch duct IPMN (88\% and $67 \%$, respectively) [10]. Interestingly, according to the current survey, despite the lack of international recommendations, cholangioscopy was more frequently used for delineation of CCA (52.3\%), with $93.3 \%$ of these respondents using it as part of the preoperative workup, compared with pancreatoscopy for delineation of IPMN (46.1\%), where $80 \%$ used it as preoperative work-up. Also noteworthy is that approximately half of the respondents that used IPOC in preoperative work-up ( $42.2 \%$ and $46.4 \%$, respectively) reported that the surgical approach was altered in $21 \%-40 \%$ of patients, based on preoperative cholangioscopic or pancreatoscopic findings. This seems to be a considerable proportion of patients and is in line with the results of two recently published studies $[11,12]$. Tyberg et al. reported that the surgical approach was altered based on preoperative IPOC in $34 \%$ of patients in total. In 8/13 patients with IPMN, the surgical approach changed: less extensive surgery in 4 patients and more extensive surgery in 4 patients. Furthermore, in 32/105 patients with presumed CCA, the surgical approach changed: less extensive surgery in 6 patients and avoidance of surgery in 26 patients, 14 of whom were found to have benign disease and 12 of whom were determined to be irresectable [11]. Pereira et al. reported that the anatomical classification of CCA was altered based on cholangioscopic findings in $42 \%$ of patients, resulting in a changed surgical approach in $21 \%(n=4)$ : more extensive surgery in 2 patients and avoidance of surgery due to irresectability in 2 patients [12]. In both studies, a combination of visual assessment of the lesions and biopsy findings was used for preoperative assessment and led to identification of benign disease or a more extensive intraductal disease. Given the relatively high proportion of patients in whom the surgical approach was altered, this could be a very promising indication for IPOC, both to aid in the differentiation of cancerous from noncancerous lesions and for determination of lesion extent. However, given the risk of inducing cholangitis or post-ERCP pancreatitis preoperatively, possibly postponing surgical treatment in these patients, a decision to perform cholangiopancreatoscopy should be made after multidisciplinary discussion in a team with radiologists, surgeons, and gastroenterologists. In these two previous studies, the rates of cholangitis or postERCP pancreatitis were $0-7 \%$ and $2.5 \%-11.6 \%$, respectively $[11,12]$. Currently, data on preoperative use of IPOC for CCA and IPMN are still scarce, and therefore investigating the clinical utility and adverse event rate of preoperative delineation in prospective studies might be useful to determine the exact role, as it could lower the treatment burden for patients and lead to decreased health care costs for the treatment of patients with CCA or IPMN.

Another important finding of this survey involved the role of pancreatoscopy-guided lithotripsy in the treatment algorithm of symptomatic PD stones. Although the ESGE guideline recommends ESWL as first-line therapy for PD stones of $>5 \mathrm{~mm}$, and to consider pancreatoscopic lithotripsy in cases of ESWL failure [13], 77.9\% of our experienced endoscopists performed pancreatoscopy-guided lithotripsy without prior ESWL. Although there is no prospective literature available on the technical and clinical success of pancreatoscopy with EHL or laser lithotripsy as first-line treatment in patients with intraductal stones, very high success rates have been reported as second-line therapy [14]. The advantages of performing this treatment are that successful fragmentation can be achieved in a mean of two lithotripsy procedures [14] and that the strictures can be treated simultaneously. Compared with ESWL, it also allows not only stone fragmentation but also stone fragment removal at the same time. This could result in a lower total number of procedures, thereby reducing patient burden and costs. However, prospective studies are needed to investigate the technical and clinical success of pancreatoscopy-guided lithotripsy as a first-line treatment option, ideally followed by a randomized controlled trial directly comparing EHL or laser lithotripsy with ESWL.

Similarly to pancreatoscopy-guided lithotripsy, a considerable proportion of the respondents (30.4\%) reported using cholangioscopy-guided lithotripsy as a first-line treatment option in selected cases without prior ERCP with conventional extraction techniques (i.e. basket or balloon). Again, this is not recommended by the ESGE guideline and cholangioscopy-guided lithotripsy is only recommended in cases of difficult bile duct stones [15]. Although not specified in this survey, "selected cases" might be patients with one or multiple risk factors for difficult bile duct stone removal. As second-line treatment after ERCP with conventional extraction techniques, cholangioscopyguided lithotripsy has shown promising results regarding technical and clinical success ( $99 \%$ and $92 \%$, respectively) for treatment of difficult bile duct stones in a recently published study by Minami et al. [16]. To the best of our knowledge, there are no studies available on technical and clinical success of IPOC-guided lithotripsy as a first-line treatment option. An advantage of this treatment strategy might be to prevent patients from undergoing repeated ERCP procedures without successful removal, with concomitant risk of ERCP-related complications. However, a diagnostic algorithm should be conducted to select the most appropriate patients who might benefit from a cholangioscopy-first treatment, and subsequently technical and clinical success needs to be investigated.

A striking finding was that, even though the majority of respondents administered intravenous antibiotics during the investigation, $23.3 \%$ did not. A retrospective study by Sethi et al. found a higher rate of cholangitis in patients undergoing ERCP with IPOC compared with patients undergoing ERCP without IPOC [17]. More recently, Thosani et al. found that bacteremia was specifically related to IPOC, and not ERCP, in $13.9 \%$ of pa- 
tients [18]. Othman et al. also found bacteremia to be present in $9 \%$ of patients post-IPOC, with a higher rate of bacteremia in patients in whom biopsy sampling was performed compared with patients without sampling $(P=0.011)$ [19]. A definitive explanation for increased bacteremia and cholangitis has not been established, but the necessity of fluid irrigation (saline or water), perhaps into poorly drained biliary segments, under increased ductal pressure, is likely to play an important role. Given the increased risk of bacteremia and cholangitis, the ESGE technology review has already stated that administration of antibiotics in patients undergoing IPOC is considered to be important [3], and therefore it would also be vital that this is included in an international clinical practice guideline to standardize use of antibiotic prophylaxis in this subgroup of patients undergoing ERCP.

Finally, also noteworthy is that there was considerable variation in the type of sedation used for ERCP without IPOC and ERCP with IPOC. For ERCP without IPOC the most common type of sedation used was almost equally divided between conscious sedation, nurse-administered propofol sedation or propofol sedation administered by an anesthesiologist, and general anesthesia with endotracheal intubation. However, when ERCP was performed with IPOC, propofol administered by an anesthesiologist or general anesthesia with endotracheal intubation were the two most commonly used types of sedation ( $27.9 \%$ and $40.7 \%$, respectively). This may be due to the fact that IPOC procedures are often lengthy and may be technically difficult to perform. Administration of enhanced anesthesia possibly makes IPOC procedures more comfortable for patients, which in turn makes the procedure easier for the endoscopist to perform.

A strength of this study is that we were able to reach a wide variety of endoscopists in many different countries. Unfortunately, in many countries we received a completed survey from only one endoscopist. Therefore, we were not able to analyze the difference in use between countries and regions. However, we were able to provide a general overview of the clinical practice patterns of IPOC throughout Europe. Another limitation of the survey is that strict privacy laws restricted our ability to contact endoscopists directly to invite them to participate in the survey. For example, we were not permitted to directly contact SpyGlass users who had given consent to Boston Scientific Inc. for commercial use of their contact details and were dependent on distribution of the survey through local representatives. This could have led to the low response rate $(23.3 \%)$, which could be seen as a limitation of this paper with regard to providing a general overview of European clinical practice. However, we were able to collect responses from experienced endoscopists and therefore deem our results representative of current clinical practice. Nevertheless, it is not possible to be certain from this survey whether the study respondents represent usual IPOC practice in Europe.

In conclusion, this first European survey on clinical practice patterns of IPOC provides an overview of current clinical use of IPOC. The results demonstrated consensus in its role for the most common indications (assessment of biliary strictures and management of difficult biliary stones), but considerable varia- tion in its overall role and in areas of clinical practice (e. g. pancreatic disease). There is an urgent need for standardization of indications and technical performance in an international consensus position statement.

\section{Acknowledgments}

We thank all endoscopists who completed this survey. The European Cholangioscopy Group has received an unrestricted grant from Boston Scientific Inc.

\section{Competing interests}

George Webster is a consultant and invited speaker for Boston Scientific, Cook Endoscopy, and Pentax Medical. Mark Ellrichmann has received consulting and lecture fees from Boston Scientific. Marco Bruno is a consultant for and has received grants for industry- and investigator-initiated studies from Boston Scientific, Cook Medical, and Pentax Medical; he has also received grants for investigator-initiated studies from Mylan, Interscope, and $3 \mathrm{M}$. Vincenzo Cennamo is a consultant for and has received speaker fees and travel grants from Olympus Italia, Olympus Europa, Euromedical, and Novità Medicali. The remaining authors declare that they have no conflict of interest.

References

[1] Ghersi S, Fuccio L, Bassi M et al. Current status of peroral cholangioscopy in biliary tract diseases. World J Gastrointest Endosc 2015; 7: 510-517

[2] Komanduri S, Thosani N. ASGE Technology Committee. et al. Cholangiopancreatoscopy. Gastrointest Endosc 2016; 84: 209-221

[3] Tringali A, Lemmers A, Meves V et al. Intraductal biliopancreatic imaging: European Society of Gastrointestinal Endoscopy (ESGE) technology review. Endoscopy 2015; 47: 739-753

[4] de Oliveira P, de Moura DTH, Ribeiro IB et al. Efficacy of digital singleoperator cholangioscopy in the visual interpretation of indeterminate biliary strictures: a systematic review and meta-analysis. Surg Endosc 2020; 34: 3321-3329

[5] Sethi A, Tyberg A, Slivka A et al. Digital single-operator cholangioscopy (DSOC) improves interobserver agreement (IOA) and accuracy for evaluation of indeterminate biliary strictures: the Monaco classification. J Clin Gastroenterol 2020: doi:10.1097/MCG. 0000000000001321

[6] Kalaitzakis E, Webster G], Oppong KW et al. Diagnostic and therapeutic utility of single-operator peroral cholangioscopy for indeterminate biliary lesions and bile duct stones. Eur J Gastroenterol Hepatol 2012; 24: 656-664

[7] Gerges C, Beyna T, Tang RSY et al. Digital single-operator peroral cholangioscopy-guided biopsy sampling versus ERCP-guided brushing for indeterminate biliary strictures: a prospective, randomized, multicenter trial (with video). Gastrointest Endosc 2020; 91: 11051113

[8] Varadarajulu S, Bang JY, Hasan MK et al. Improving the diagnostic yield of single-operator cholangioscopy-guided biopsy of indeterminate biliary strictures: ROSE to the rescue? (with video) Gastrointest Endosc 2016; 84: 681-687

[9] Ali S, Hawes RH, Kadkhodayan K et al. Utility of rapid onsite evaluation of touch imprint cytology from endoscopic and cholangioscopic forceps biopsy sampling (with video). Gastrointest Endosc 2019; 89: $340-344$ 
[10] European Study Group on Cystic Tumours of the Pancreas. European evidence-based guidelines on pancreatic cystic neoplasms. Gut 2018; 67: 789-804

[11] Tyberg A, Raijman I, Siddiqui A et al. Digital pancreaticocholangioscopy for mapping of pancreaticobiliary neoplasia: can we alter the surgical resection margin? J Clin Gastroenterol 2019; 53: 71-75

[12] Pereira P, Santos S, Morais R et al. Role of peroral cholangioscopy for diagnosis and staging of biliary tumors. Dig Dis 2020; 38: 431-440

[13] Dumonceau JM, Delhaye M, Tringali A et al. Endoscopic treatment of chronic pancreatitis: European Society of Gastrointestinal Endoscopy (ESGE) Guideline - updated August 2018. Endoscopy 2019; 51: 179193

[14] McCarty TR, Sobani Z, Rustagi T. Per-oral pancreatoscopy with intraductal lithotripsy for difficult pancreatic duct stones: a systematic review and meta-analysis. Endosc Int Open 2020; 8: E1460-E1470

[15] Manes G, Paspatis G, Aabakken L et al. Endoscopic management of common bile duct stones: European Society of Gastrointestinal Endoscopy (ESGE) guideline. Endoscopy 2019; 51: 472-491
[16] Minami H, Mukai S, Sofuni A et al. Clinical outcomes of digital cholangioscopy-guided procedures for the diagnosis of biliary strictures and treatment of difficult bile duct stones: a single-center large cohort study. J Clin Med 2021; 10: 1638

[17] Sethi A, Chen YK, Austin GL et al. ERCP with cholangiopancreatoscopy may be associated with higher rates of complications than ERCP alone: a single-center experience. Gastrointest Endosc 2011; 73: 251-256

[18] Thosani N, Zubarik RS, Kochar R et al. Prospective evaluation of bacteremia rates and infectious complications among patients undergoing single-operator choledochoscopy during ERCP. Endoscopy 2016; 48: $424-431$

[19] Othman MO, Guerrero R, Elhanafi S et al. A prospective study of the risk of bacteremia in directed cholangioscopic examination of the common bile duct. Gastrointest Endosc 2016; 83: 151-157 